metallic iron present in them formed by the reduction of magnetite by carbon.

A previous report ${ }^{17}$ of presolar Ne-E in a carbon-rich residue from one of the ordinary chondrites studied by Scott et al. led them to suspect that the graphitemagnetite inclusions might be the carrier, and hence be of presolar origin. Unfortunately the story is not that simple - they did not find any Ne-E in the graphitemagnetite intergrowths. Thus the Ne-E must either be contained in a different carbonaceous component, or in another minor phase in the residue. The identification of the carriers of such minor gas components is fraught with difficulties, but is providing us with valuable clues to the early history of the Solar System.

\title{
Impact cratering
}

\section{from Richard A.F. Grieve}

The relative importance of cratering in planetary evolution was evidenced at the Twelfth Lunar and Planetary Science Conference* by numerous papers on impact craters and discussion, in sessions dealing with particular terrestrial planets and the icy satellites of Jupiter and Saturn, of their effects. The diversity of research reported in the sessions dealing directly with impact processes reflected the random nature of cratering with respect to specific geological environments and the involvement of many sub-disciplines of the physical science in providing information on cratering processes. As has been apparent in recent years, there was an increasing combination of theoretical and observational results, with each approach serving to constrain the other.

With respect to the evolution of the jovian and saturnian satellites, the results of impact experiments showed that the mechanical properties of ice differ from those of rock, even at the low temperatures of $70-80 \mathrm{~K}$ existing on these bodies. In particular, the specific energy required to disrupt an icy body by impact appears to be -20 per cent less than for a silicate body. Other impact experiments illustrated that extensive fracturing occurs around craters in ice but craters in ice-saturated sand targets exhibit more limited fracturing and are more like those in competent rock. It was noted, however, that ejection patterns appear to be similar for craters in icy and silicate targets.

Computational simulations derived from the solution of continuum mechanics equations continued to provide important insights into cratering processes. For example, they indicated that excavation dynamics and the physical state and radial distribution of ejecta are strong functions of impact velocity. Calculations also supported the interpretation that bowlshaped cavities are produced by excavation plus the displacement of the cavity floor by impact-induced flow fields. Further confirmation of these displacements was proved by an analysis of the Cactus nuclear event. Similar conclusions on the formation of bowl-shaped craters were reached in a

*The Twelfith Lunar and Planetary Science Conterence was held in Houston, Texas in March.

combined observational and theoretical treatment of the Brent Crater, Canada, which also provided an estimate of the initial impact conditions: a $113-\mathrm{m}$ radius chondritic projectile impacting a $\approx 27 \mathrm{~km}$ $\mathrm{s}^{-1}$.

The important question of the detailed particle flow fields created in the target rocks by impact was addressed in a comparison of flow fields derived from continuum mechanics calculations and the 'Maxwell-Z' model. It was clear that this empirically derived model remains a powerful analytical tool. Similarities between the calculated flow fields for Meteor Crater, Arizona, and those of the Middle Gust III explosive event also suggested that the parametric codes derived from the latter may be applicable to impact events. However, the energy coupling differences suggest that explosive events are imperfect analogues to impact events in terms of scaling crater dimensions with energy.

It is known that the initial transient cavities formed by impact events generally modify rapidly to more stable final forms. In bowl-shaped craters, this modification is thought to occur by failure of the transient cavity walls. It was proposed at the meeting, however, that cavity modification may result from the centripetal motion of the entire rim area, in a manner similar to that suggested for the formation of larger, relatively shallow complex impact structures. The formation of these complex structures was addressed in a phenomenological model, ascribing central peak and ring formation to oscillatory motions of the transient cavity floor. These oscillatory motions require essentially plastic behaviour and viscosity of $10^{9} \mathrm{P}$ for the target rocks. Such viscosities may result from the effective fluidization of fractured target by an impact-generated acoustic field. Some support for the importance of two parameters, rock strength and gravity, in the phenomenological model may be found in the report that the $20-\mathrm{km}$ Haughton impact structure on Devon Island, Canada, has a multi-ring form. Haughton was formed in sediments and its relatively small dimensions for a ring structure scale with gravity and rock strength when compared with similar crater forms on the Moon and in crystalline targets on Earth. Doubts were raised, however, over the universal concept of deep initial transient cavities by the results of impact experiments at high gravitational accelerations, which suggested that relatively shallow cavities may be produced directly without modification.

Several papers dealt with the mineralogical or shock metamorphic effects produced by impacts in various target media. Shock experiments in limestone indicated that incipient vapourization of carbonate occurs at pressures as low as $350 \mathrm{kbar}$. An analysis of effects in tectosilicates illustrated that planar deformation features are produced during shock compression. It was also reported that the reduction in refractive index of shocked quartz is due to lattice disordering. A high concentration of lattice defects, particularly non-bridging oxygens, is also the cause of the conversion of plagioclase to the solid-state glassy phase known as maskelynite at shock pressures of 300-350 kbar.

The non-isochemical alteration of impact melt rocks was reported, as was evidence for extensive chemical exchange between feldspar clasts and enclosing impact melt matrix. This chemical exchange may account for some of the disturbances seen in the Rb-Sr systematics of melt rocks. ${ }^{39} \mathrm{Ar}-{ }^{40} \mathrm{Ar}$ systematics of shocked samples from the Ries, Germany, were also described and it appears that up to 99 per cent of radiogenic ${ }^{40} \mathrm{Ar}$ may be lost at shock pressures of $300 \mathrm{kbar}$ or more. This observation should aid in the interpretation of Ar ages obtained on lunar impact breccias.

A report of siderophile abundances in the melt rocks at Strangways suggested that this 20-25- $\mathrm{km}$ impact structure in Australia was formed by an olivine-rich achondrite. Siderophile enrichments were also discussed with reference to the Cretaceous-Tertiary boundary layer and extinctions. It was noted that several important geochemical problems with respect to the various sampled sections need to be resolved. Theoretical calculations were presented for the postulated extinction-causing impact and indicated that significant atmospheric heating could only be achieved indirectly through energy transfer from ejecta. The interpretation that the formation of the $20-\mathrm{km}$ lunar crater Giordano Bruno is recorded in the Gervase of Canterbury chronicles as occurring on 19 June 1178 was reviewed. It appears that only an estimated 0.1 per cent of the ejecta might reach the Earth by direct trajectories and that the analytical problems associated with recognizing such material are formidable.

Richard A.F. Grieve is Visiting Professor at the Department of Geological Sciences, Brown University, Rhode Island. 